\title{
Residual-Based Simpler Block GMRES for Nonsymmetric Linear Systems with Multiple Right-Hand Sides
}

\author{
Qinghua $W u\left(\mathbb{D},{ }^{1,2}\right.$ Liang Bao, ${ }^{3}$ and Yiqin Lin $\mathbb{D}^{2}$ \\ ${ }^{1}$ School of Mathematics and Computational Science, Xiangtan University, Xiangtan 411105, China \\ ${ }^{2}$ College of Science, Hunan University of Science and Engineering, Yongzhou 425100, China \\ ${ }^{3}$ Department of Mathematics, East China University of Science and Technology, Shanghai 200237, China
}

Correspondence should be addressed to Qinghua Wu; jackwqh@163.com

Received 16 November 2017; Accepted 12 March 2018; Published 16 April 2018

Academic Editor: Soheil Salahshour

Copyright (C) 2018 Qinghua Wu et al. This is an open access article distributed under the Creative Commons Attribution License, which permits unrestricted use, distribution, and reproduction in any medium, provided the original work is properly cited.

\begin{abstract}
We propose in this paper a residual-based simpler block GMRES method for solving a system of linear algebraic equations with multiple right-hand sides. We show that this method is mathematically equivalent to the block GMRES method and thus equivalent to the simpler block GMRES method. Moreover, it is shown that the residual-based method is numerically more stable than the simpler block GMRES method. Based on the deflation strategy proposed by Calandra et al. (2013), we derive a deflation strategy to detect the possible linear dependence of the residuals and a near rank deficiency occurring in the block Arnoldi procedure. Numerical experiments are conducted to illustrate the performance of the new method.
\end{abstract}

\section{Introduction}

In this paper, we consider iterative methods for solving a system of linear algebraic equations:

$$
A X=B,
$$

where $A$ is a nonsingular matrix of order $n$ and $X=$ $\left[x_{1}, \ldots, x_{s}\right]$ and $B=\left[b_{1}, \ldots, b_{s}\right]$ are rectangular matrices of dimension $n \times s$ with $s \leq n$. For solving such systems, the block GMRES [1] and its variants are very popular. Block GMRES is based on the block Arnoldi process and is formally fully analogous to the ordinary GMRES algorithm by Saad and Schultz [2].

The following notation is used throughout the paper. Subscripts denote the iteration index and superscripts distinguish between individual columns in a block. We denote by $\|\cdot\|$ the Euclidean vector norm and the induced matrix norm and by $\|A\|_{F}$ the Frobenius norm. Moreover, for $A \in \mathbb{R}^{N \times n}(N>n)$ of rank $n, \kappa(A)=\sigma_{1}(A) / \sigma_{n}(A)$ is the spectral condition number, where $\sigma_{1}(A)>\sigma_{n}(A)>0$ are the extremal singular values of $A$.
Given an initial approximation $X_{0} \in \mathbb{R}^{n \times s}$ to the solution of (1), let

$$
R_{0}=B-A X_{0},
$$

and then in analogy to the unblocked case, we build a sequence of iterates $X_{m} \in X_{0}+K_{m}\left(A, R_{0}\right)$ such that

$$
\left\|R_{m}\right\|_{F}=\left\|B-A X_{m}\right\|_{F}=\min _{\widetilde{X} \in X_{0}+K_{m}\left(A, R_{0}\right)}\left\|R_{0}-A \widetilde{X}\right\|_{F},
$$

where $K_{m}\left(A, R_{0}\right)=\operatorname{span}\left\{R_{0}, A R_{0}, \ldots, A^{m-1} R_{0}\right\}$. Equation (3) is equivalent to minimizing every column of $R_{m}$, that is,

$$
\begin{aligned}
& \left\|R_{m}^{(i)}\right\|_{2}=\min \\
& \quad \text { subject to } X_{m}^{(i)}-X_{0}^{(i)} \in K_{m}\left(A, R_{0}\right),
\end{aligned}
$$

and also to the orthogonality condition

$$
R_{m}^{(i)} \perp A K_{m}\left(A, R_{0}\right), \quad i=1, \ldots, s,
$$

where $\perp$ is the orthogonality relation induced by the Euclidean inner product. Assume that $X_{m}$ is of the form 
(1) Given $R_{0}=B-A X_{0}$ of full rank.

(2) Compute the QR factorization of $R_{0}: R_{0}=Y_{1} \rho_{0}$.

(3) For $j=1, \ldots, m$

(3.1) Compute $W=A Y_{j}$.

(3.2) For $i=1, \ldots, j$

$$
H_{i, j}=Y_{i}^{T} W \text {. }
$$$$
W=W-Y_{i} H_{i, j} \text {. }
$$

End For

(3.3) Compute the QR factorization of $W: W=Y_{j} H_{j+1, j}$. End For

Algorithm 1: Nondeflated block Arnoldi process.

$X_{m}=X_{0}+\mathscr{Y}_{m} t_{m}$, where $\mathscr{Y}_{m}=\left[Y_{1}, Y_{2}, \ldots, Y_{m}\right]$ is a basis of $K_{m}\left(A, R_{0}\right)$. Then, we obtain the $m$ th residual matrix

$$
R_{m}=R_{0}-A \mathscr{Y}_{m} t_{m} .
$$

Central to the usual implementations of block GMRES is the block Arnoldi process [1], which can be used to construct the orthonormal basis of $K_{m}\left(A, R_{0}\right)$. In practice, the possible linear dependence of the residuals of the $s$ systems requires an explicit reduction of the number of right-hand sides. In [3], this was called deflation. If the block residual is nearly rank deficient, block GMRES should be implemented with deflation and there are various sophisticated rank-revealing QR factorizations. For details, see [3] and the references therein. We can write the nondeflated block Arnoldi process as shown in Algorithm 1.

From Algorithm 1, we obtain formally the ordinary Arnoldi relation

$$
A \mathscr{Y}_{m}=\mathscr{Y}_{m+1} \mathscr{H}_{m}
$$

where the $(m+1) s \times m s$ matrix is

$$
\mathscr{H}_{m}:=\left[\begin{array}{cccc}
H_{1,1} & H_{1,2} & \cdots & H_{1, m} \\
H_{2,1} & H_{2,2} & \cdots & H_{2, m} \\
& H_{3,2} & \ddots & \vdots \\
& & \ddots & H_{m, m} \\
& & & H_{m+1, m}
\end{array}\right] .
$$

In the block Arnoldi algorithm, $Y_{i}^{T} Y_{i}=I$ holds due to the QR factorizations, and $Y_{i}^{T} Y_{j}=0$ when $i \neq j$, where $I$ is a unit matrix and 0 is a zero matrix of order $s$. This indicates that the whole process is equivalent to the one in which the block vectors are generated column by column using an ordinary modified Gram-Schmidt process.

From (6) and (7), we obtain the fundamental block GMRES relation

$$
R_{m}=\mathscr{Y}_{m+1}\left(e_{1} \rho_{0}-\mathscr{H}_{m} t_{m}\right),
$$

where $e_{1}$ is the first $s$ column of the $(m+1) s \times(m+1) s$ unit matrix (the size changes with $m$ ), $\rho_{0}$ is an upper triangular matrix obtained in Arnoldi's initialization step, and $t_{m}$ is the "block coordinates" of $X_{m}-X_{0}$ with respect to the block Arnoldi basis.

Using (9), the least squares problem (3) is solved by recursive QR factorization of $\mathscr{H}_{m}$, updated by applying Givens rotations. Once the norm of the residual is small enough, the triangular system with the computed $R$-factor is solved, and the approximate solution $X_{m}$ is computed. The detailed algorithm of block GMRES can be found in [3-5].

The block GMRES method with deflation at each iteration was proposed in [6]. And a deflation strategy was investigated to detect when a linear combination of approximate solutions is already known; for details, see [7]. In this paper, we deal with a different approach and compare the situation with deflation and without deflation. Let $\mathscr{Z}_{m}$ be a block basis of $K_{m}\left(A, R_{0}\right)$. Instead of building a block orthogonal basis of $K_{m}\left(A, R_{0}\right)$, we look for a block orthogonal basis $\mathscr{V}_{m}=\left[V_{1}, \ldots, V_{m}\right]$ of $A K_{m}\left(A, R_{0}\right)$. As a special case, simpler block GMRES (SBGMRES) was proposed by Liu and Zhong [8], where $\mathscr{Z}_{m}=$ $\left[R_{0} /\left\|R_{0}\right\|_{F}, V_{1}, \ldots, V_{m-1}\right]$. In this paper, we will consider the basis $\mathscr{Z}_{m}=\left[R_{0} /\left\|R_{0}\right\|_{F}, R_{1} /\left\|R_{1}\right\|_{F}, \ldots, R_{m-1} /\left\|R_{m-1}\right\|_{F}\right]$. We call this case the residual-based simpler block GMRES (RBSBGMRES).

The paper is organized as follows. In Section 2, the RBSBGMRES and SBGMRES algorithms are described (without deflation). In Section 3, some comparison between RBSBGMRES and SBGMRES is established. In Section 4, the RB-SBGMRES method with deflation and the corresponding algorithm RB-SBGMRES-D (Table 2) are derived. In Section 5, these algorithms are compared using test matrices taken from the Matrix Market [9]. Conclusions are included in the last section.

\section{Residual-Based Simpler Block GMRES}

Suppose that $\mathscr{Z}_{m} \equiv\left[Z_{1}, \ldots, Z_{m}\right]$ is a basis of $K_{m}\left(A, R_{0}\right)$. The orthogonal basis $\mathscr{V}_{m}$ of $A K_{m}\left(A, R_{0}\right)$ is thus obtained from the QR factorization of $A \mathscr{Z}_{m}$; that is,

$$
A \mathscr{Z}_{m}=\mathscr{V}_{m} \mathscr{U}_{m},
$$

where $\mathcal{U}_{m}$ is an upper triangular matrix with order $m s$.

Due to the orthogonality property $R_{m} \perp A K_{m}\left(A, R_{0}\right)$, the $m$ th residual matrix $R_{m}$ can be computed recursively as

$$
R_{m}=R_{0}-\mathscr{V}_{m}\left(\mathscr{V}_{m}^{T} R_{0}\right)=R_{m-1}-V_{m} S_{m}
$$

where $S_{m}=V_{m}^{T} R_{m-1}$.

Define $D_{m}=\left[S_{1}^{T}, \ldots, S_{m}^{T}\right]^{T}$. Since the columns of $\mathscr{Z}_{m}$ form a basis of $K_{m}\left(A, R_{0}\right)$, we can represent $X_{m}$ in the form

$$
X_{m}=X_{0}+\mathscr{E}_{m} t_{m}
$$

where $t_{m} \in R^{m s \times s}$ is the "block coordinates" of $X_{m}-X_{0}$ with respect to the block basis $\mathscr{Z}_{m}$. Due to $R_{m}=R_{0}-A \mathscr{Z}_{m} t_{m}$, $A \mathscr{E}_{m}=\mathscr{V}_{m} \mathscr{U}_{m}$, and $R_{m} \perp A K_{m}\left(A, R_{0}\right)$, it follows that

$$
\mathcal{U}_{m} t_{m}=\mathscr{V}_{m}^{T} R_{0}=D_{m}
$$


(1) Given $X_{0}$, set $R_{0}=B-A X_{0}$. If $\left\|R_{0}\right\|_{F} \leq$ TOL, accept $X_{0}$ and exit.

(2) For $j=1, \ldots, m$

(2.1) Compute $W_{j}=A R_{j-1}$.

(2.2) For $i=1, \ldots, j-1$

$$
U_{i j}=V_{i}^{T} W_{j} \text {. }
$$

End For

$W_{j}=W_{j}-V_{i} U_{i j}$.

(2.3) Compute the QR factorization of $W_{j}: W_{j}=V_{j} U_{j j}$.

(2.4) Compute $S_{j}=V_{j}^{T} R_{j-1}, R_{j}=R_{j-1}-V_{j} S_{j}$.

(2.5) If $\left\|R_{j}\right\|_{F}<$ TOL, break.

End For

(3) Solve the triangular system $\mathcal{U}_{j} t_{j}=D_{j}$ for $t_{j}$.

(4) From the approximate solution

$$
X_{j}=X_{0}+\left[R_{0}, \ldots, R_{j-1}\right] t_{j} .
$$

(5) If $\left\|R_{j}\right\|_{F}<$ TOL, then accept $X_{j}$ and exit; otherwise, restart: set $X_{0}=X_{j}$ and go to Step (1).

Algorithm 2: RB-SBGMRES(m).

(1) Given $X_{0}$, set $R_{0}=B-A X_{0}$. If $\left\|R_{0}\right\|_{F} \leq$ TOL, accept $X_{0}$ and exit.

(2) For $j=1, \ldots, m$

(2.1) Compute $W_{j}=A V_{j-1}$ if $j>1, W_{1}=A R_{0}$ for $j=1$.

(2.2) For $i=1, \ldots, j-1$

$$
\begin{aligned}
& U_{i j}=V_{i}^{T} W_{j} . \\
& W_{j}=W_{j}-V_{i} U_{i j} .
\end{aligned}
$$

End For

(2.3) Compute the QR factorization of $W_{j}: W_{j}=V_{j} U_{j j}$.

(2.4) Compute $S_{j}=V_{j}^{T} R_{j-1}, R_{j}=R_{j-1}-V_{j} S_{j}$.

(2.5) If $\left\|R_{j}\right\|_{F}<$ TOL, break.

End For

(3) Solve the triangular system $\mathscr{U}_{j} t_{j}=D_{j}$ for $t_{j}$.

(4) Form the approximate solution

$$
X_{j}=X_{0}+\left[R_{0}, V_{1}, \ldots, V_{j-1}\right] t_{j} .
$$

(5) If $\left\|R_{j}\right\|_{F}<$ TOL, then accept $X_{j}$ and exit; otherwise, restart: set $X_{0}=X_{j}$ and go to Step (1).

Algorithm 3: SBGMRES(m).

Hence, once the residual norm is small enough, we can solve this upper triangular system (13) and then compute the approximate solution $X_{m}$.

We now present the RB-SBGMRES method without deflation as shown in Algorithms 2 and 3 and Table 1. For comparison, we also present the SBGMRES method proposed in [8].

\section{Comparison with SBGMRES}

In [8], an equivalence between SBGMRES and classical block GMRES had been established. Algorithm 2 indicates that RBSBGMRES is equivalent to block GMRES; that is, search a solution $X_{m} \in K_{m}\left(A, R_{0}\right)$, such that $R_{m} \perp A K_{m}\left(A, R_{0}\right)$.

On the other hand, for single right-hand side, it has been observed in [10] that the gap between the true residual $R T_{m}=B-A X_{m}$ and the updated residual $R_{m}$ can be strongly influenced by the conditioning of $\mathscr{Z}_{m}$, which is the basis of $K_{m}\left(A, R_{0}\right)$, and the choice of the basis $\mathscr{X}_{m}$ has an effect on the conditioning of the matrix $\mathscr{U}_{m}$ [10]. Since we compute
TABLE 1: Computational cost of a cycle of RB-SBGMRES (or SBGMRES).

\begin{tabular}{lc}
\hline Step & Computational cost \\
\hline Computation of $R_{0}$ & $n s$ \\
Computation of $W_{j}$ & $n s$ \\
Block Arnoldi procedure (2.1)-(2.2) & $O\left(n s^{2}\right)$ \\
QR factorization of $W_{j}$ & $2 n s^{2}+n s$ \\
Computation of $S_{j}, R_{j}$ & $2 s n$ \\
Computation of $t_{j}$ & $O\left(j s^{3}\right)$ \\
Computation of $X_{j}$ & $n j s$ \\
\hline
\end{tabular}

the coordinates of the correction $X_{m}-X_{0}$ in the basis $\mathscr{Z}_{m}$ by (13), the approximate solution $X_{m}$ becomes inaccurate as the conditioning of $\mathscr{U}_{m}$ grows. Simpler GMRES [11] is, in general, less accurate than GMRES and is inherently unstable due to the choice of the basis $\left[R_{0}, V_{1}, \ldots, V_{m-1}\right]$. It is easy to formulate an analogous conclusion in the block case. 
TABLE 2: Computational cost of a cycle of RB-SBGMRES-D (Algorithm 4).

\begin{tabular}{lc}
\hline Step & Computational cost \\
\hline Computation of $R_{0}$ & $n s$ \\
Computation of $\mathscr{G}_{0}$ & $14 s^{3}$ \\
Computation of $W_{j}$ & $n c_{j}$ \\
Computation of $\mathscr{F}_{1}$ & $14 s^{3}$ \\
Block Arnoldi procedure (5.3) & $O\left(n k_{j}^{2}\right)$ \\
Computation of $\left[V_{j}, P_{j-1}\right]$ & $2 n s^{2}$ \\
QR factorization of $R_{0}$ & $2 n s^{2}+n s$ \\
Computation of $\left[Z_{j+1}, Q_{j}\right]$ & $2 n s^{2}$ \\
Computation of $X_{j}$ & $n \sum_{i=1}^{j} c_{i}$ \\
QR factorization of $R_{0}$ & $2 n s^{2}+n s$ \\
Computation of $\left[Z_{1}, Q_{0}\right]$ & $2 n s^{2}$ \\
QR factorization of $W_{1}$ & $2 n c_{1}^{2}+n c_{1}$ \\
Computation of $\left[V_{1}, P_{0}\right]$ & $2 n s^{2}$ \\
Computation of $\mathscr{F}_{j}$ & $4 s_{j} s^{2}+14 s^{3}$ \\
Computation of $S_{j}, R_{j}$ & $2 k_{j} n$ \\
Computation of $\mathscr{G}_{j}$ & $4 s_{j} s^{2}+14 s^{3}$ \\
Computation of $t_{j}$ & $O\left(\sum_{i=1}^{j} c_{i}\right)^{3}$ \\
\hline
\end{tabular}

There is a theorem about condition number of $\left[R_{0} /\left\|R_{0}\right\|_{F}, V_{1}, \ldots, V_{m-1}\right]$ in [8] stated as follows.

Theorem 1. For $\mathscr{Z}_{m}=\left[R_{0} /\left\|R_{0}\right\|_{F}, V_{1}, \ldots, V_{m-1}\right]$, one has

$$
\kappa\left(\mathscr{Z}_{m}\right) \leq \frac{2 s^{1 / 2} \kappa\left(R_{m-1}\right)\left\|R_{0}\right\|_{F}}{\left\|R_{m-1}\right\|_{F}},
$$

where $s$ is the number of right-hand sides.

The condition number of $\mathscr{Z}_{m}=\left[R_{0} /\left\|R_{0}\right\|_{F}, \ldots\right.$, $\left.R_{m-1} /\left\|R_{m-1}\right\|_{F}\right]$ may have an effect on the conditioning of the matrix $\mathscr{U}_{m}$. In the following, we formulate an analogous theorem on the condition number of $\mathscr{Z}_{m}$.

Theorem 2. Suppose that $m$ steps of RB-SBGMRES have been taken and $R_{i} v=R_{i-1} v, i=1, \ldots, m-1$, if and only if $v$ is a zero vector; then, one has

$$
\begin{aligned}
& \kappa\left(\mathscr{Z}_{m}\right) \\
& \leq \sqrt{m\left(s \kappa\left(R_{m-1}\right)^{2}+\sum_{i=1}^{m-1} \frac{\left(\left\|R_{i-1}\right\|_{F}^{2}+\left\|R_{i}\right\|_{F}^{2}\right)}{\min _{\|v\|=1}\left(\left\|R_{i-1} v\right\|^{2}-\left\|R_{i} v\right\|^{2}\right)}\right)},
\end{aligned}
$$

where $\mathscr{Z}_{m}=\left[R_{0} /\left\|R_{0}\right\|_{F}, \ldots, R_{m-1} /\left\|R_{m-1}\right\|_{F}\right]$ and $s$ is the number of right-hand sides.

Proof. Let $R_{m-1}=\bar{Q}_{m-1} \bar{R}_{m-1}$ be a QR factorization of $R_{m-1}$. We have

$$
\begin{aligned}
\mathscr{Z}_{m} & =\left[\frac{R_{0}}{\left\|R_{0}\right\|_{F}}, \ldots, \frac{R_{m-1}}{\left\|R_{m-1}\right\|_{F}}\right] \\
& =\left[V_{1}, \ldots, V_{m-1}, \bar{Q}_{m-1}\right] C_{m}
\end{aligned}
$$

where

$$
C_{m}=\left[\begin{array}{cccc}
\frac{S_{1}}{\left\|R_{0}\right\|_{F}} & 0 & \cdots & 0 \\
\frac{S_{2}}{\left\|R_{0}\right\|_{F}} & \frac{S_{2}}{\left\|R_{1}\right\|_{F}} & \cdots & \\
\vdots & \vdots & \vdots & \vdots \\
\frac{S_{m-1}}{\left\|R_{0}\right\|_{F}} & \frac{S_{m-1}}{\left\|R_{1}\right\|_{F}} & \cdots & 0 \\
\frac{\pi_{m-1}}{\left\|R_{0}\right\|_{F}} & \frac{\bar{R}_{m-1}}{\left\|R_{1}\right\|_{F}} & \cdots & \frac{\bar{R}_{m-1}}{\left\|R_{m-1}\right\|_{F}}
\end{array}\right] .
$$

Moreover, we get $\left\|R_{m-1}\right\|_{F}=\left\|\bar{R}_{m-1}\right\|_{F}$.

From (11), we obtain

$$
\left\|R_{i}\right\|_{F}^{2}=\sum_{j=i}^{m-1}\left\|S_{j}\right\|_{F}^{2}+\left\|R_{m-1}\right\|_{F}^{2} .
$$

Then, it follows that

$$
\left\|C_{m}\right\|_{F}=\sqrt{m}
$$

Since the columns of $\left[V_{1}, \ldots, V_{m-1}, \bar{Q}_{m-1}\right]$ are orthogonal, it follows that

$$
\kappa\left(\mathscr{Z}_{m}\right)=\kappa\left(C_{m}\right) .
$$

On the other hand,

$$
C_{m}^{-1}=\left[\begin{array}{cccccc}
S_{1}^{-1}\left\|R_{0}\right\|_{F} & 0 & 0 & \cdots & 0 & 0 \\
-S_{1}^{-1}\left\|R_{1}\right\|_{F} & S_{2}^{-1}\left\|R_{1}\right\|_{F} & 0 & \cdots & 0 & 0 \\
0 & -S_{2}^{-1}\left\|R_{2}\right\|_{F} & \ddots & \cdots & 0 & 0 \\
\vdots & \vdots & \ddots & \ddots & \vdots & \vdots \\
0 & 0 & 0 & \ddots & S_{m-1}^{-1}\left\|R_{m-2}\right\|_{F} & 0 \\
0 & 0 & 0 & \cdots & -S_{m-1}^{-1}\left\|R_{m-1}\right\|_{F} & \bar{R}_{m-1}^{-1}\left\|R_{m-1}\right\|_{F}
\end{array}\right]
$$


Thus, using the triangle inequality and the fact that the 1norm is bounded from above by the 2-norm, we have

$$
\begin{aligned}
\left\|C_{m}^{-1}\right\|^{2} \leq & \left\|\bar{R}_{m-1}^{-1}\right\|^{2}\left\|R_{m-1}\right\|_{F}^{2} \\
& +\sum_{i=1}^{m-1}\left(\left\|R_{i-1}\right\|_{F}^{2}+\left\|R_{i}\right\|_{F}^{2}\right)\left\|S_{i}^{-1}\right\|^{2} .
\end{aligned}
$$

The norm of $S_{i}^{-1}$ can be expressed using (11) as

$$
\begin{aligned}
\left\|S_{i}^{-1}\right\|^{2} & =\frac{1}{\min _{\|v\|=1}\left\|S_{i} v\right\|^{2}} \\
& =\frac{1}{\min _{\|v\|=1}\left(\left\|R_{i-1} v\right\|^{2}-\left\|R_{i} v\right\|^{2}\right)} .
\end{aligned}
$$

With $\left\|R_{m-1}\right\|_{F} \leq \sqrt{s}\left\|R_{m-1}\right\|$, we have

$$
\begin{aligned}
\left\|C_{m}^{-1}\right\|^{2} \leq & s \kappa\left(R_{m-1}\right)^{2} \\
& +\sum_{i=1}^{m-1} \frac{\left(\left\|R_{i-1}\right\|_{F}^{2}+\left\|R_{i}\right\|_{F}^{2}\right)}{\min _{\|v\|=1}\left(\left\|R_{i-1} v\right\|^{2}-\left\|R_{i} v\right\|^{2}\right)}
\end{aligned}
$$

The proof then follows from $\left\|C_{m}\right\|_{2} \leq\left\|C_{m}\right\|_{F}=\sqrt{m}$.

Theorem 1 indicates that the conditioning of $\left[R_{0} /\left\|R_{0}\right\|_{F}, \mathscr{V}_{m-1}\right]$ is inversely proportional to the actual relative norm of the residual. Once residuals become small, this will lead to the ill-conditioning of the matrices $\mathscr{Z}_{m}$ and the matrices $\mathscr{U}_{m}$, and SBGMRES will behave unstably after some initial residual reduction. However, from Theorem 2, the conditioning of $\left[R_{0} /\left\|R_{0}\right\|_{F}, \ldots, R_{m-1} /\left\|R_{m-1}\right\|_{F}\right]$ is related to the intermediate decrease of the residual norms, not to the residual decrease with respect to the initial residual. For single right-hand sides, it has been observed that $\left[R_{0} /\left\|R_{0}\right\|_{F}, \ldots, R_{m-1} /\left\|R_{m-1}\right\|_{F}\right]$ remains well conditioned while $\left[R_{0} /\left\|R_{0}\right\|_{F}, \mathscr{V}_{m-1}\right]$ becomes ill-conditioned [10].

\section{RB-SBGMRES with Deflation}

When block Krylov subspace methods are used for the solution of linear systems of equations with multiple righthand sides, the linear dependence of the residual of the $s$ systems may occur, and this is called deflation. Deflation may be possible at startup or in a later step. Sometimes, we need to incorporate a strategy for detecting when a linear combination of systems has approximately converged. Recently, Calandra et al. derived a deflation strategy to detect a near rank deficiency occurring in the block Arnoldi procedure in [7]. We provide a brief overview of the method.

Assume that the QR factorization of $R_{0}$ has been performed as

$$
R_{0}=\widehat{\mathscr{Y}}_{1} \widehat{\rho}_{0},
$$

with $\widehat{\mathscr{Y}}_{1} \in R^{n \times s}$ having orthonormal columns and $\hat{\rho}_{0} \in R^{s \times s}$. To circumvent deflation at startup, the subspace decomposition at the beginning of the cycle is derived by finding a unitary matrix $\mathscr{F}_{1} \in R^{s \times s}$, such that

$$
\left[\mathscr{Y}_{1}, P_{0}\right]=\widehat{\mathscr{Y}}_{1} \mathscr{F}_{1}
$$

with $\mathscr{Y}_{1} \in R^{n \times k_{1}}, P_{0} \in R^{n \times d_{1}}$, and $k_{1}+d_{1}=s$.

The unitary matrix $\mathscr{F}_{1}$ is determined by the singular value decomposition (SVD) of $\widehat{\rho}_{0}=U \Sigma W^{T}$ and set $\mathscr{F}_{1}=U$. Choose a relative deflation threshold $\varepsilon$ and select $k_{1}$ singular values of $\hat{\rho}_{0}$ such that $\sigma\left(\hat{\rho}_{0}\right)>\varepsilon$.

Define $q_{0}=0$ and $q_{j}=q_{j-1}+k_{j}$, for $\left[\mathscr{Y}_{j}, P_{j-1}\right] \epsilon$ $R^{n \times\left(q_{j}+d_{j}\right)}$ with orthonormal columns, and assume that the following block Arnoldi relation holds at the beginning of the jth iteration:

$$
A \mathscr{Y}_{j-1}=\left[\mathscr{Y}_{j}, P_{j-1}\right] \mathscr{H}_{j-1}, \quad j>1,
$$

with $\mathscr{Y}_{j-1} \in R^{n \times q_{j-1}}, \mathscr{Y}_{j} \in R^{n \times q_{j}}, P_{j-1} \in R^{n \times d_{j}}$, and $\mathscr{H}_{j-1} \in R^{\left(q_{j-1}+p\right) \times q_{j-1}}$. The $j$ th iteration of the deflated block Arnoldi procedure produces matrices $\widehat{Y}_{j+1} \in R^{n \times k_{j}}$ and $\widehat{\mathscr{H}}_{j} \in$ $R^{\left(q_{j}+p\right) \times q_{j}}$ which satisfy

$$
A\left[\mathscr{Y}_{j-1}, Y_{j}\right]=\left[\mathscr{Y}_{j}, P_{j-1}, \widehat{Y}_{j+1}\right] \widehat{\mathscr{H}}_{j},
$$

with $\widehat{\mathscr{H}}_{j}$ having the following block structure:

$$
\widehat{\mathscr{H}}_{j}=\left(\begin{array}{cc}
\mathscr{H}_{j-1} & H_{j} \\
0_{k_{j} \times q_{j-1}} & H_{j+1, j}
\end{array}\right) .
$$
lated as

Defining $\widehat{\mathscr{Y}}_{j+1}=\left[\mathscr{Y}_{j}, P_{j-1}, \widehat{Y}_{j+1}\right],(28)$ can be reformu-

$$
A \mathscr{Y}_{j}=\widehat{\mathscr{Y}}_{j+1} \widehat{\mathscr{H}}_{j-1} \text {. }
$$

The subspace decomposition is performed by finding a unitary matrix $\mathscr{F}_{j+1}$ such that

$$
\left[\mathscr{Y}_{j+1}, P_{j}\right]=\widehat{\mathscr{Y}}_{j+1} \mathscr{F}_{j+1} .
$$

Hence, we obtain

$$
A \mathscr{Y}_{j}=\widehat{\mathscr{Y}}_{j+1} \mathscr{F}_{j+1} \mathscr{F}_{j+1}^{T} \widehat{\mathscr{H}}_{j-1} .
$$

Defining $\mathscr{H}_{j} \in R^{\left(q_{j}+p\right) \times q_{j}}$ as $\mathscr{H}_{j}=\mathscr{F}_{j+1} \widehat{\mathscr{H}}_{j}$, this leads to

$$
A \mathscr{Y}_{j}=\left[\mathscr{Y}_{j+1}, P_{j}\right] \mathscr{H}_{j} \text {, }
$$

which is the block Arnoldi relation required at the beginning of the next iteration.

From (31), the unitary matrix $\mathscr{F}_{j+1}$ has the following matrix structure:

$$
\mathscr{F}_{j+1}=\left(\begin{array}{cc}
I_{q_{j}} & 0_{q_{j} \times p} \\
0_{p \times q_{j}} & F_{j}
\end{array}\right) .
$$


Defining $\widehat{\rho}_{j} \in R^{\left(q_{j}+p\right) \times p}$ as $\widehat{\rho}_{j}=\left(\begin{array}{c}\mathscr{F}_{j}^{T} \widehat{\rho}_{j-1} \\ 0_{k_{j} \times p}\end{array}\right)$ and $\widehat{\mathscr{R}}_{j}=\widehat{\rho}_{j}-\widehat{\mathscr{H}}_{j} Y_{j}$ with $\widehat{\mathscr{R}}_{0}=\widehat{\rho}_{0}$, the unitary matrix $\mathscr{F}_{j+1}$ is determined by the following steps (for details, see [7]):

(1) SVD of $\widehat{\mathscr{R}}_{j}=U \Sigma W^{T}$, with $U=\left(\begin{array}{c}U_{q_{j}}^{+} U_{q_{j}}^{-} \\ U_{s}^{+} U_{s}^{-}\end{array}\right)$.

(2) QR decomposition of $s \times s$ matrix $\left[U_{s}^{+} U_{s}^{-}\right]$.

In the case of simpler block GMRES method, the relationship $R_{j}=\widehat{\mathscr{Y}}_{j+1} \widehat{\mathscr{R}}_{j}$, which is an important ingredient for the block GMRES method, cannot be established. We must find another strategy to perform the subspace decomposition.

Assume that the $\mathrm{QR}$ factorization of $R_{0}$ has been performed as

$$
R_{0}=\widehat{Z}_{1} \widehat{\rho}_{0} .
$$

We compute $\mathscr{G}_{0}$ analogously as $\mathscr{F}_{1}$ in (26), which leads to the following formulas:

$$
\begin{aligned}
{\left[Z_{1}, Q_{0}\right] } & =\widehat{Z}_{1} \mathscr{G}_{0}, \\
R_{0} & =\left[Z_{1}, Q_{0}\right] \rho_{0},
\end{aligned}
$$

with $\rho_{0}=\mathscr{G}_{0}^{T} \hat{\rho}_{0}, Z_{1} \in R^{n \times c_{1}}$ and $Q_{0} \in R^{n \times l_{1}}, c_{1}+l_{1}=s$. To build a block orthogonal basis of $A K_{m}\left(A, R_{0}\right)$, we compute the QR factorization of $A Z_{1}$ :

$$
\begin{aligned}
& A Z_{1}=\widehat{\mathscr{V}}_{1} \widehat{\mathscr{U}}_{1}, \\
& A Z_{1}=\left[\mathscr{V}_{1}, P_{0}\right] \mathscr{U}_{1},
\end{aligned}
$$

with $\left[\mathscr{V}_{1}, P_{0}\right]=\widehat{\mathscr{V}}_{1} \mathscr{F}_{1}, \widehat{\mathscr{V}}_{1} \in R^{n \times c_{1}}, V_{1}=\mathscr{V}_{1}, \widehat{\mathscr{U}}_{1} \in R^{c_{1} \times c_{1}}$, $P_{0} \in R^{n \times d_{1}}$, and $k_{1}+d_{1}=c_{1}$, and $\mathscr{F}_{1} \in R^{c_{1} \times c_{1}}$ is computed similarly to $\mathscr{G}_{0}$.

Assume that the block Arnoldi relation

$$
A \mathscr{Z}_{j}=\left[\mathscr{V}_{j}, P_{j-1}\right] \mathscr{U}_{j},
$$

holds at the beginning of the $(j+1)$ th iteration of the deflated block Arnoldi procedure, with $Z_{j} \in R^{n \times c_{j}}, V_{j} \in R^{n \times k_{j}}, P_{j-1} \in$ $R^{n \times d_{j}}, \mathscr{U}_{j} \in R^{q_{j} \times q_{j}}, \mathscr{Z}_{j}=\left[Z_{1}, \ldots, Z_{j}\right]$, and $\mathscr{V}_{j}=\left[V_{1}, \ldots, V_{j}\right]$, where $q_{j}, s_{j}$, and $k_{j}$ are defined as follows: $q_{1}=c_{1}, q_{j}=q_{j-1}+$ $c_{j}, s_{1}=k_{1}$, and $s_{j}=s_{j+1}=s_{j}+k_{j+1}$.

Set $R_{j}=R_{j-1}-V_{j} S_{j}$, with $V_{j} \in R^{n \times k_{j}}$, and define $S_{j} \in$ $R^{k_{j} \times s}$ as $S_{j}=V_{j}^{T} R_{j-1}$. We generate $Z_{j+1}$ by calculating the QR factorization of $R_{j}$, which leads to the following formulas:

$$
\begin{aligned}
R_{j} & =\widehat{Z}_{j+1} \widehat{\rho}_{j} \\
& =\left[Z_{j+1}, Q_{j}\right] \rho_{j},
\end{aligned}
$$

with $Z_{j+1} \in R^{n \times c_{j+1}}, Q_{j} \in R^{n \times l_{j+1}}, c_{j+1}+l_{j+1}=s,\left[Z_{j+1}, Q_{j}\right]=$ $\widehat{Z}_{j+1} \mathscr{G}_{j}$, and $\rho_{j}=\mathscr{G}_{j}^{T} \widehat{\rho}_{j}$, and $\mathscr{G}_{j}$ is a unitary matrix, which is determined similarly to $\mathscr{G}_{0}$.

Compute $A Z_{j+1}$ and make it orthogonal to the columns of $\left[\mathscr{V}_{j}, P_{j-1}\right]$ by an ordinary modified Gram-Schmidt process, such that

$$
A\left[\mathscr{Z}_{j}, Z_{j+1}\right]=\left[\mathscr{V}_{j}, P_{j-1}, \widehat{V}_{j+1}\right] \widehat{\mathscr{U}}_{j+1},
$$

with $\widehat{V}_{j+1} \in R^{n \times c_{j+1}}$, and $\widehat{\mathscr{U}}_{j+1} \in R^{q_{j+1} \times q_{j+1}}$ have the following block structure:

$$
\widehat{\mathscr{U}}_{j+1}=\left(\begin{array}{cc}
\mathcal{U}_{j} & U_{j+1} \\
0 & U_{j+1, j+1}
\end{array}\right)=\left(\begin{array}{cc}
\mathcal{U}_{j}^{+} & U_{j+1}^{+} \\
\mathcal{U}_{j}^{-} & U_{j+1}^{-} \\
0 & U_{j+1, j+1}
\end{array}\right),
$$

where $U_{j+1} \in R^{\left(s_{j}+d_{j}\right) \times c_{j+1}}, s_{j}+d_{j}=q_{j}, U_{j+1, j+1} \in R^{c_{j+1} \times c_{j+1}}$, $U_{j+1}^{+} \in R^{s_{j} \times c_{j+1}}$, and $U_{j+1}^{-} \in R^{d_{j} \times c_{j+1}}$. Define $\Lambda_{j+1} \in R^{\left(d_{j}+c_{j+1}\right) \times q_{j}}$ as

$$
\Lambda_{j+1}=\left(\begin{array}{cc}
\mathcal{U}_{j}^{-} & U_{j+1}^{-} \\
0 & U_{j+1, j+1}
\end{array}\right) .
$$

We formulate

$$
\left[V_{j+1}, P_{j}\right]=\left[P_{j-1}, \widehat{V}_{j+1}\right] \mathscr{F}_{j+1},
$$

by calculating the SVD of $\Lambda_{j+1}=U \Sigma W^{T}$, instead of computing the SVD of $\mathscr{R}_{j}$. Note that $U \in R^{\left(d_{j}+c_{j+1}\right) \times\left(d_{j}+c_{j+1}\right)}$; we set $\mathscr{F}_{j+1}=U$ and select $k_{j+1}$ singular values of $\Lambda_{j+1}$ such that $\sigma\left(\Lambda_{j+1}\right)>\varepsilon$. Once a near rank deficiency occurs in the block Arnoldi procedure, it will be reflected by the singular values of $\Lambda_{j+1}$. We now present the RB-SBGMRES method with deflation as shown in Algorithms 4 and 5.

Denote by $k_{j, m}$ and $c_{j, m}$ the number of Krylov directions keeping at the $j$ th iteration of the $m$ th cycle; Algorithm 4 indicates that $k_{j+1, m}<k_{j, m}$ and $c_{j+1, m}<c_{j, m}$.

\section{Numerical Experiments}

In this section, RB-SBGMRES is tested and compared with SBGMRES. The test matrices were taken from the Matrix Market [9]. All computations were carried out using Matlab on a PC with the usual double precision, where the floating point relative accuracy is $2.22 \times 10^{-16}$. In the following examples, we take $X_{0}$ as the zero matrix; thus, the initial residual matrix is $R_{0}=B-A X_{0}=B$, and we set $h=\pi / N$, where $N$ is the order of the matrix $A$. We plot the relative true norm of residual

$$
\frac{\left\|R T_{m}\right\|_{F}}{\left\|R_{0}\right\|_{F}}=\frac{\left\|B-A X_{m}\right\|_{F}}{\left\|B-A X_{0}\right\|_{F}}=\frac{\left\|B-A X_{m}\right\|_{F}}{\|B\|_{F}}
$$

and condition number of $\mathcal{U}_{m}$, respectively, in Figures 1,2 , and 3. The condition number of $\mathcal{U}_{m}$ is computed by Matlab internal function cond.

Example 1. The matrix is fs1836 $\left(\|A\| \approx 1.2 \cdot 10^{9}, \kappa(A) \approx 1.5\right.$. $10^{11}$ ) which is a matrix of order 183 , and we have $s=2$ righthand sides which are chosen as

$$
\begin{aligned}
& B(:, 1)=A \cdot e, \\
& B(:, 2)=A \cdot(\sin (h), \sin (2 h), \ldots, \sin (N h))^{T},
\end{aligned}
$$

where $B(:, m)$ is the $m$ th column of matrix $B, h=\pi / N$, and $e \equiv[1,1, \ldots, 1]^{T}$. 
(1) Given $X_{0} \in R^{n \times s}$, set $R_{0}=B-A X_{0}$. If $\left\|R_{0}\right\|_{F} \leq$ TOL, accept $X_{0}$ and exit.

(2) Compute the QR factorization of $R_{0}: R_{0}=\widehat{Z}_{1} \widehat{\rho}_{0}$.

(3) Determine deflation unitary matrix $\mathscr{G}_{0} \in R^{s \times s}$ and $c_{1}, l_{1}$ such that $c_{1}+l_{1}=s$ (see Algorithm 5), and set $q_{1}=c_{1}$.

(4) Define $\left[Z_{1}, Q_{0}\right]=\widehat{Z}_{1} \mathscr{G}_{0}$, with $Z_{1} \in R^{n \times c_{1}},\left(Q_{0} \in R^{n \times l_{1}}\right)$ as the first columns of $\widehat{Z}_{1} \mathscr{G}_{0}$, and define $Z_{1}=\mathscr{Z}_{1}$.

(5) For $j=1, \ldots, m$

(5.1) Compute $W_{j}=A Z_{j}$.

(5.2) If $j=1$

(5.2.1) Compute the QR factorization of $W_{j}: W_{j}=A Z_{1}=\widehat{V}_{1} \widehat{\mathscr{U}}_{1}$.

(5.2.2) Determine deflation unitary matrix $\mathscr{F}_{1} \in R^{c_{1} \times c_{1}}$ and $k_{1}, d_{1}$ such that $k_{1}+d_{1}=c_{1}$ (see Algorithm 5), and set $s_{1}=k_{1}$.

(5.2.3) Define $\left[V_{1}, P_{0}\right]=\widehat{V}_{1} \mathscr{F}_{1}$, with $V_{1} \in R^{n \times k_{1}},\left(P_{0} \in R^{n \times d_{1}}\right)$ as the first columns of $\widehat{V}_{1} \mathscr{F}_{1}$, and define $V_{1}=\mathscr{V}_{1}$.

(5.3) Else

(5.3.1) For $i=1, \ldots, j-1$

$$
U_{i, j}=V_{i}^{T} W_{j}
$$

End For

$W_{j}=W_{j}-V_{i} U_{i, j}$

(5.3.2) $U_{p}=P_{j-1}^{T} W_{j}$.

(5.3.3) $W_{j}=W_{j}-P_{j-1} U_{p}$.

(5.3.4) Define $U_{j}^{T}=\left[U_{1, j}, \ldots, U_{j-1, j}, U_{p}\right]^{T}$, compute the QR factorization of $W_{j}: W_{j}=\widehat{V}_{j} U_{j, j}$, leading to

$A \mathscr{Z}_{j}=\left[\mathscr{V}_{j-1}, P_{j-2}, \widehat{V}_{j}\right] \widehat{\mathscr{U}}_{j}$, with $\widehat{\mathscr{U}}_{j} \in R^{q_{j} \times q_{j}}$ defined as (41).

(5.4) Determine deflation unitary matrix $\mathscr{F}_{j} \in R^{\left(c_{j}+d_{j-1}\right) \times\left(c_{j}+d_{j-1}\right)}$ by the SVD of $\Lambda_{j}$, and $k_{j}, d_{j}$ such that $k_{j}+d_{j}=c_{j}$ (see Algorithm 5), and set $s_{j}=s_{j-1}+k_{j}$.

(5.5) Define $\left[V_{j}, P_{j-1}\right]=\left[P_{j-2}, \widehat{V}_{j}\right] \mathscr{F}_{j}$, with $V_{j} \in R^{n \times k_{j}},\left(P_{j-1} \in R^{n \times d_{j}}\right)$ as the first columns of $\left[P_{j-2}, \widehat{V}_{j}\right] \mathscr{F}_{j}$, and set $\mathscr{U}_{j}=\left[I_{s_{j-1} \times s_{j-1}}, \mathscr{F}_{j}^{T}\right] \widehat{\mathcal{U}}_{j}, \mathscr{V}_{j}=\left[\mathscr{V}_{j-1}, V_{j}\right]$.

End If

(5.6) Compute $S_{j}=V_{j}^{T} R_{j-1}, R_{j}=R_{j-1}-V_{j} S_{j}$.

(5.7) If $\left\|R_{j}\right\|_{F}<$ TOL, break.

(5.8) Compute the QR factorization of $R_{j}: R_{j}=\widehat{Z}_{j+1} \widehat{\rho}_{j}$.

(5.9) Determine deflation unitary matrix $\mathscr{G}_{j} \in R^{s \times s}$ and $c_{j+1}, l_{j+1}$ such that $c_{j+1}+l_{j+1}=s$ (see Algorithm 5), and set $q_{j+1}=q_{j}+c_{j+1}$.

(5.10) Define $\left[Z_{j+1}, Q_{j}\right]=\widehat{Z}_{j+1} \mathscr{G}_{j}$, with $Z_{j+1} \in R^{n \times c_{j+1}},\left(Q_{j} \in R^{n \times l_{j+1}}\right)$ as the first

End For columns of $\widehat{Z}_{j+1} \mathscr{G}_{j}$, and define $\mathscr{Z}_{j+1}=\left[\mathscr{Z}_{j}, Z_{j+1}\right]$.

(6) Solve the triangular system $\mathcal{U}_{j} t_{j}=D_{j}$ for $t_{j}$, with $D_{j}=\left[S_{1}^{T}, \ldots, S_{j}^{T},\left(P_{j-1}^{T} R_{0}\right)^{T}\right]^{T}$

(7) From the approximate solution

$$
X_{j}=X_{0}+\left[Z_{1}, \ldots, Z_{j}\right] t_{j}
$$

(8) If $\left\|R_{j}\right\|_{F}<$ TOL, then accept $X_{j}$ and exit; otherwise, restart: set $X_{0}=X_{j}$ and go to Step (1).

Algorithm 4: RB-SBGMRES-D(m).

(1) Choose a relative deflation threshold $\varepsilon$.

(2) Compute the SVD of $\Lambda_{j}$ as $\Lambda_{j}=U \Sigma W^{T}$, with $U \in R^{\left(c_{j}+d_{j-1}\right) \times\left(c_{j}+d_{j-1}\right)}$, $\Sigma \in R^{\left(c_{j}+d_{j-1}\right) \times\left(c_{j}+d_{j-1}\right)}, W^{T} \in R^{\left(c_{j}+d_{j-1}\right) \times q_{j}}$; or the SVD of $\widehat{\rho}_{j}$ as $\widehat{\rho}_{j}=U_{1} \Sigma_{1} W_{1}^{T}$, with $U_{1} \in R^{s \times s}, \Sigma_{1} \in R^{s \times s}, W_{1} \in R^{s \times s}$.

(3) Select $k_{j}$ singular values of $\Lambda_{j}$, such that $\sigma_{l}\left(\Lambda_{j}\right)>\varepsilon$; or $c_{j}$ singular values of $\hat{\rho}_{j}$ such that $\sigma_{l}\left(\hat{\rho}_{j}\right)>\varepsilon$.

(4) Define $\mathscr{F}_{j}$ as $\mathscr{F}_{j}=U$; or $\mathscr{G}_{j}$ as $\mathscr{G}_{j}=U_{1}$.

Algorithm 5: Determination of $\left(k_{j}, d_{j}, \mathscr{F}_{j}\right)$ or $\left(c_{j}, l_{j}, \mathscr{G}_{j}\right)$.

In Figures 1 and 2, we show the relative true norm of residual $\left\|B-A X_{m}\right\|_{F} /\|B\|_{F}$ and condition number of $\mathscr{U}_{m}$ for the SBGMRES and RB-SBGMRES, respectively. It is clearly seen from Figure 1 that the relative true norm of residual of SBGMRES may stagnate at a significantly higher level than that of RB-SBGMRES. The reason for this is that the condition number of the matrix $\mathcal{U}_{m}$ of SBGMRES increases significantly faster than that of RB-SBGMRES as Figure 2 shows.
In Figure 3, we show the relative true norm of residual $\| B-$ $A X_{m}\left\|_{F} /\right\| B \|_{F}$ for the RB-SBGMRES-D and the BFGMRES$S$ proposed in [7], with $\varepsilon=10^{-9}$. It is clearly seen from the figure that RB-SBGMRES-D can compete with BFGMRES-S.

Example 2. The matrix is steaml $\left(\|A\| \approx 2.2 \cdot 10^{7}, \kappa(A) \approx\right.$ $3 \cdot 10^{7}$ ) which is a matrix of order 240 , and we have $s=5$ right-hand sides which are chosen as 


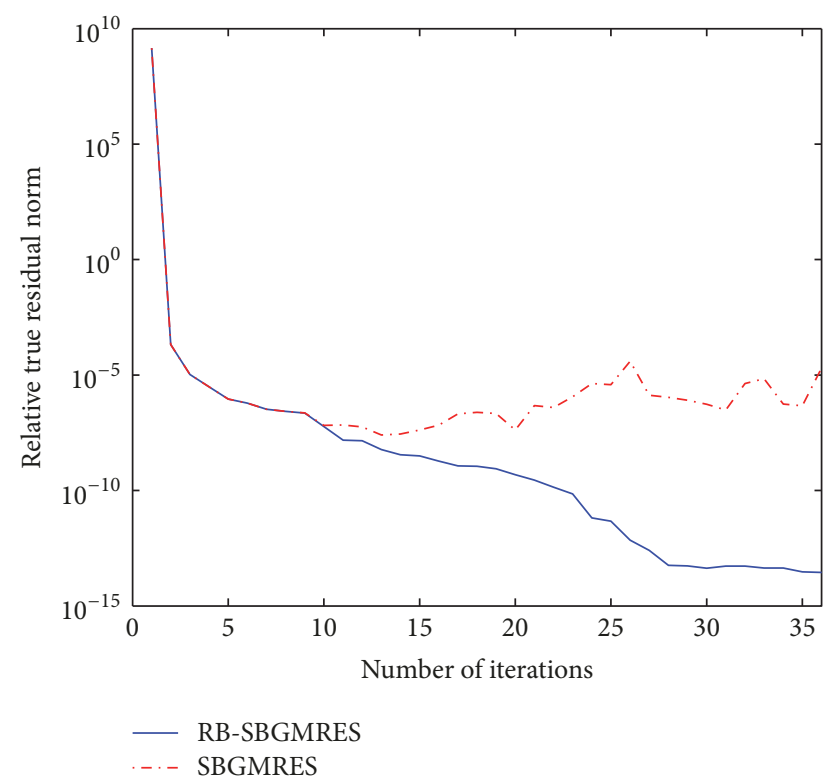

Figure 1: Example 1, the relative true residual norms versus the number of iterations.

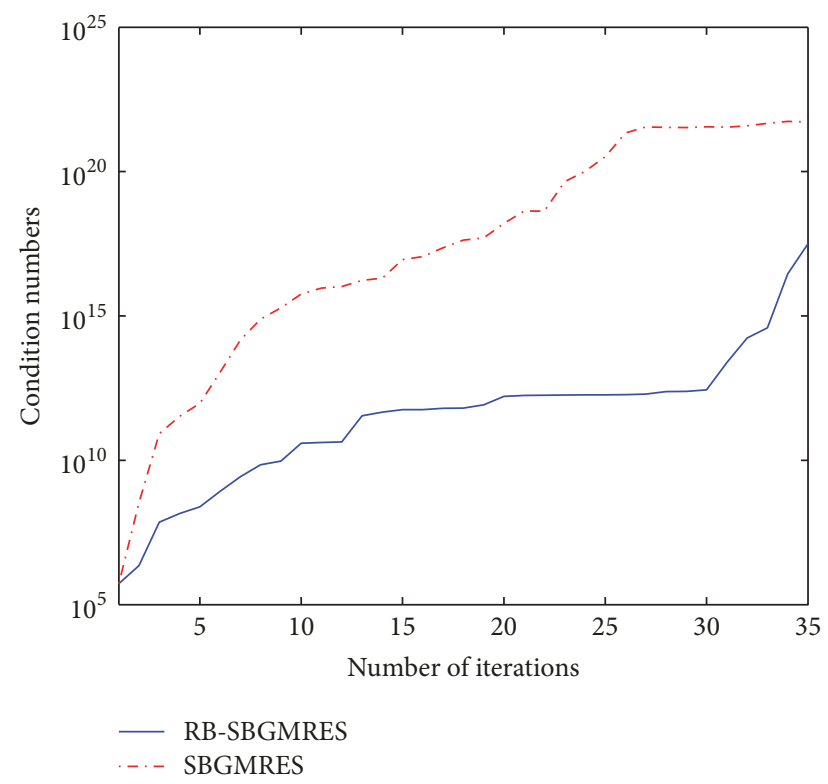

Figure 2: Example 1, the condition numbers versus the number of iterations.

$$
\begin{aligned}
& B(:, 1)=A \cdot e, \\
& B(:, 2)=A \cdot(\sin (h), \sin (2 h), \ldots, \sin (N h))^{T}, \\
& B(:, 3)=A \cdot(h, 2 h, \ldots, N h)^{T}, \\
& B(:, 4)=A \cdot(\cos (h), \cos (2 h), \ldots, \cos (N h))^{T}, \\
& B(:, 5)=A \cdot(\cos (2 h), \cos (2 h), \ldots, \cos (N h))^{T},
\end{aligned}
$$

where $B(:, m)$ is the $m$ th column of matrix $B, h=\pi / N$, and $N$ is the order of matrix $A$.

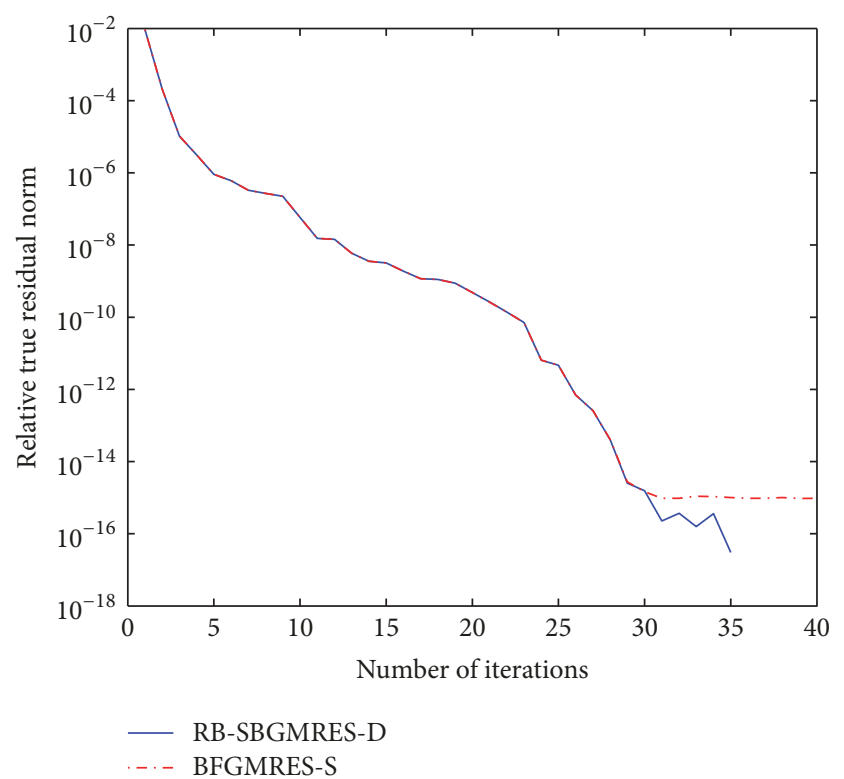

FIGURE 3: Example 1, the relative true residual norms versus the number of iterations.

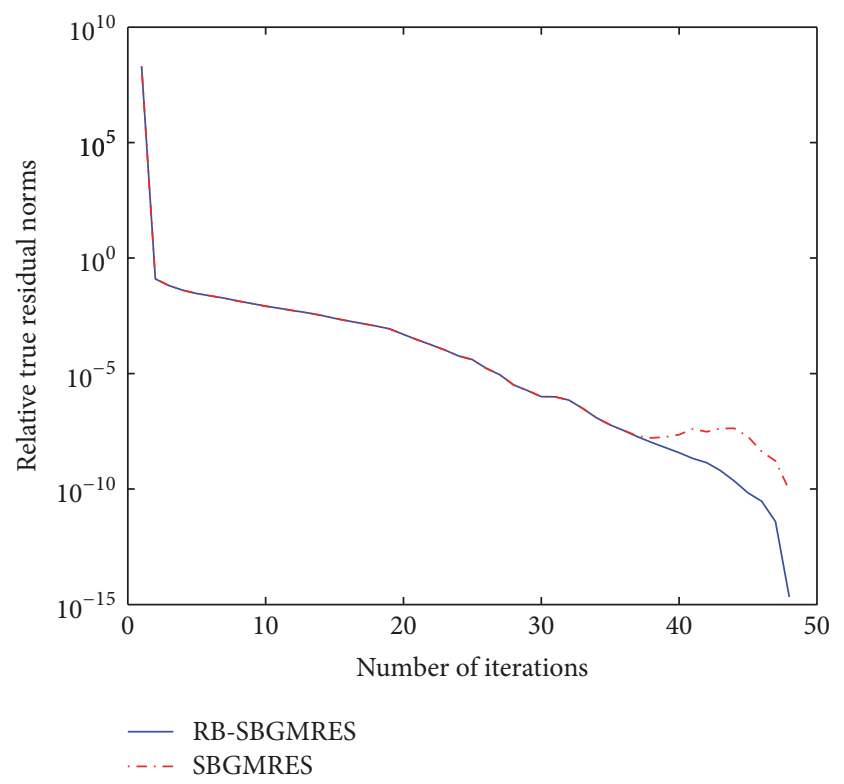

FIgURE 4: Example 2, the relative true residual norms versus the number of iterations.

It is clear from Figure 4 that the condition number of the matrix $\mathscr{U}_{m}$ of SBGMRES increases faster than that of RBSBGMRES. Since the number of right-hand sides $s=5$, the maximum iteration number is 48 . From Figure 5 , we can observe that the numerical performance of RB-SBGMRES is better than that of SBGMRES.

Figure 6 shows the relative true norm of residual $\| B-$ $A X_{m}\left\|_{F} /\right\| B \|_{F}$ for the RB-SBGMRES-D and BFGMRES-S, with $\varepsilon=10^{-9}$. It is clearly seen that the performances of RBSBGMRES-D and BFGMRES-S are almost same. 


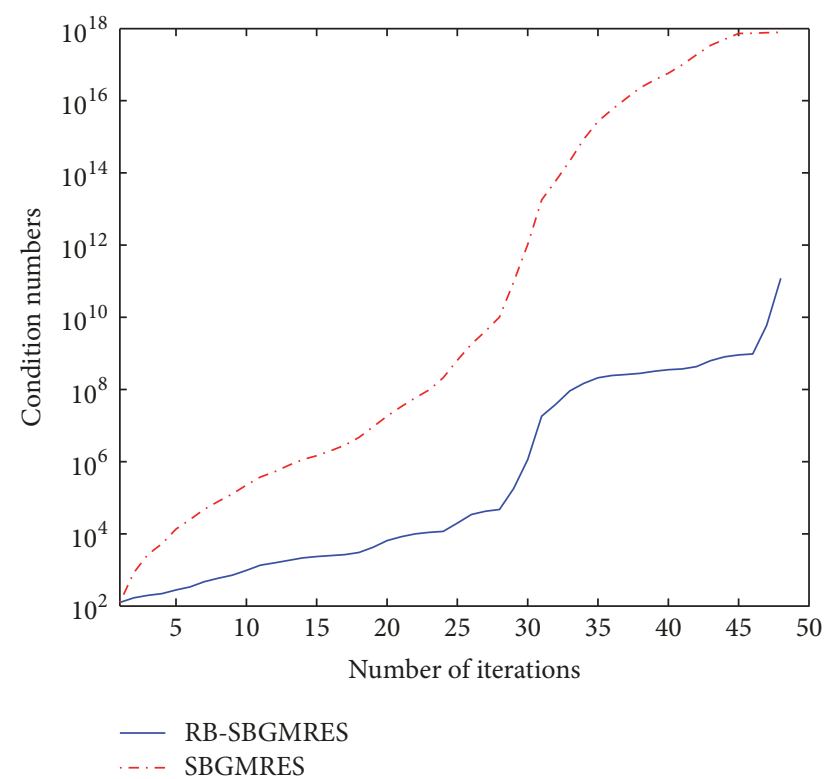

FIgURE 5: Example 2, the condition numbers versus the number of iterations.

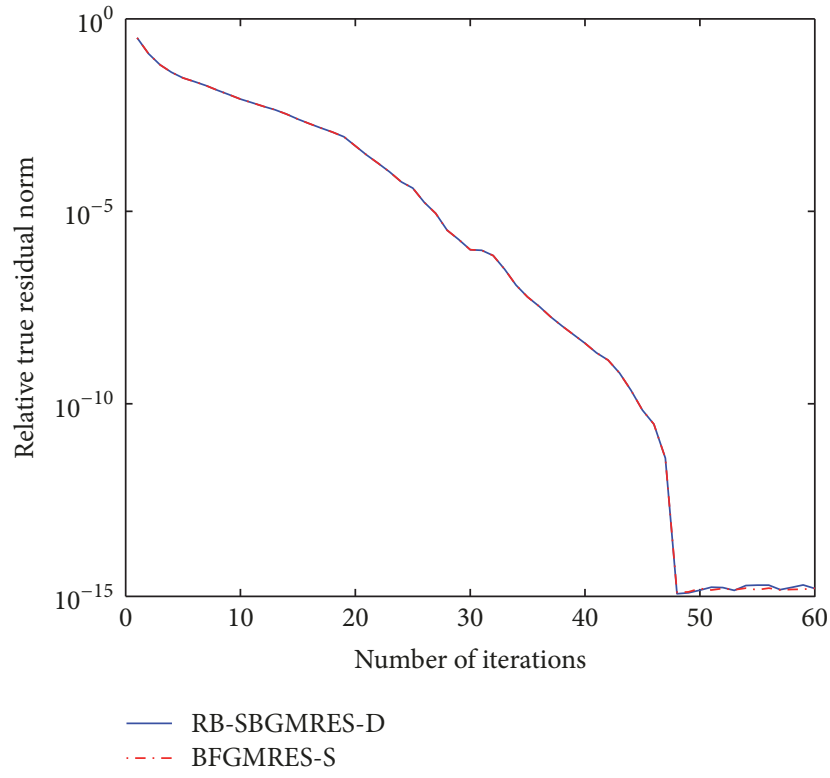

FIGURE 6: Example 2, the relative true residual norms versus the number of iterations.

Example 3. The matrix is add $20(\|A\| \approx 0.72, \kappa(A) \approx 1.76$. $10^{4}$ ) which is a matrix of order 2395, and we have $s=2$ righthand sides which are chosen as

$$
\begin{aligned}
& B(:, 1)=A \cdot e, \\
& B(i, 2)=\sin (i h), \text { for } i=1,2, \ldots, N .
\end{aligned}
$$

It is clear from Figure 7 that the numerical performance of RB-SBGMRES is better than that of SBGMRES, and from Figure 8, we see that the condition number of the matrix $\mathscr{U}_{m}$ of SBGMRES increases fast while that of RBSBGMRES remains at a significantly low level. Figure 9 shows

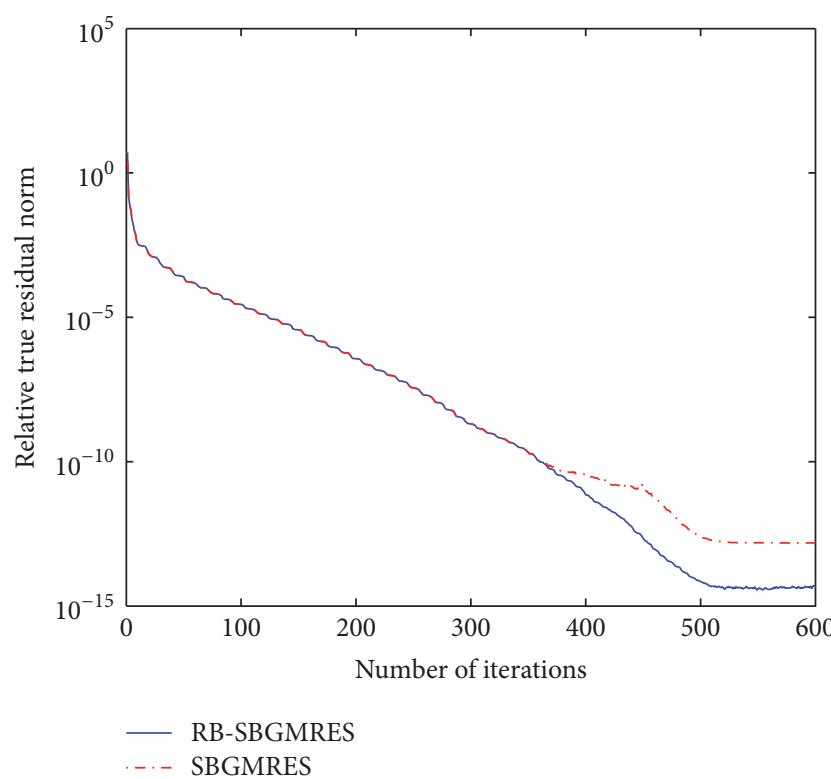

FIgURE 7: Example 3, the relative true residual norms versus the number of iterations.

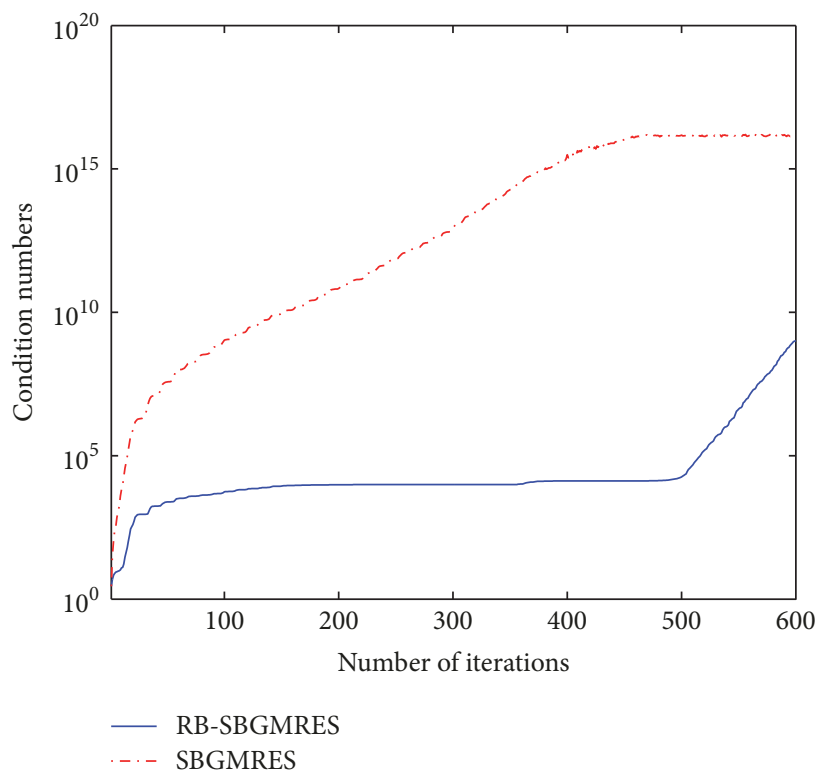

FIGURE 8: Example 3, the condition numbers versus the number of iterations.

the relative true norm of residual $\left\|B-A X_{m}\right\|_{F} /\|B\|_{F}$ for the RB-SBGMRES-D and BFGMRES-S, with $\varepsilon=10^{-15}$. The performances of RB-SBGMRES-D and BFGMRES-S are almost the same for Example 3.

Example 4. The matrix is fs $7601\left(\|A\| \approx 3 \cdot 10^{8}, \kappa(A) \approx 8.4\right.$. $10^{3}$ ) which is a matrix of order 760, and we have $s=3$ righthand sides which are chosen as

$$
\begin{aligned}
& B(:, 1)=A \cdot e, \\
& B(i, 2)=\sin (i h), \\
& B(i, 3)=i h, \text { for } i=1,2, \ldots, N .
\end{aligned}
$$




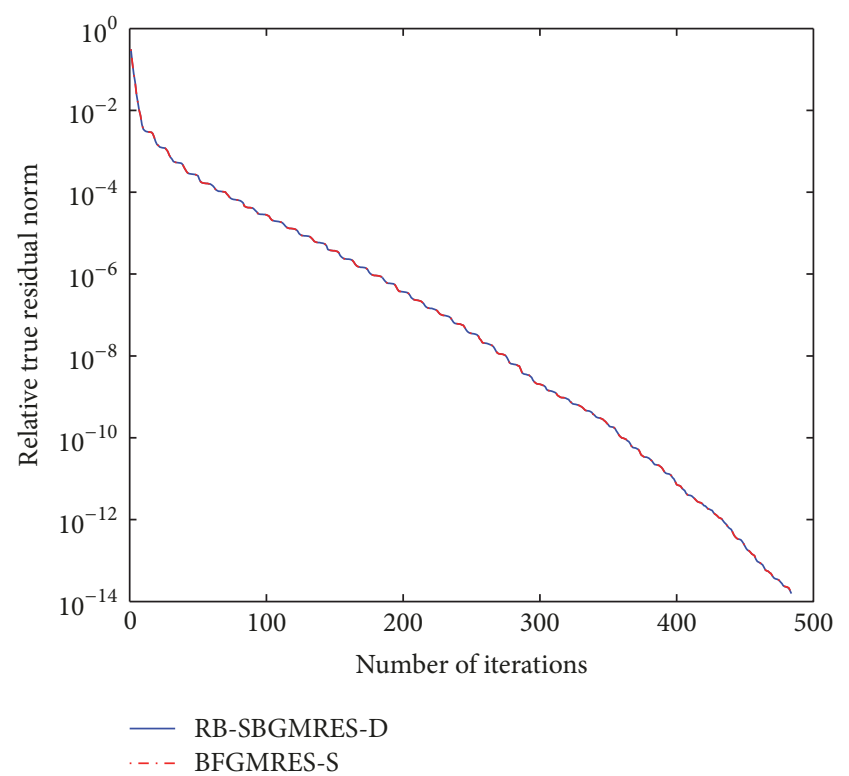

FIGURE 9: Example 3, the relative true residual norms versus the number of iterations.

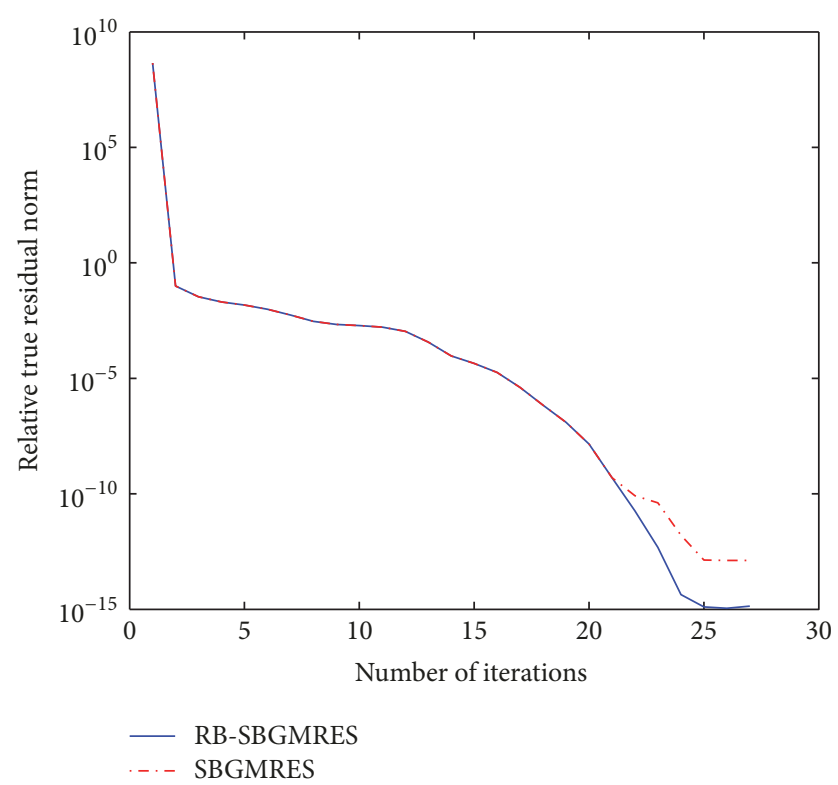

FIGURE 10: Example 4, the relative true residual norms versus the number of iterations.

It is also obvious from Figure 10 that the RB-SBGMRES method is slightly more accurate than SBGMRES in this example, and Figure 11 also shows that the condition number of the matrix $\mathscr{U}_{m}$ of SBGMRES increases faster than that of RB-SBGMRES. It is clear from Figure 12 that the performances of RB-SBGMRES-D and BFGMRES-S are almost the same for Example 4.

In order to further verify that the condition number of the matrix $\mathscr{U}_{m}$ of SBGMRES increases significantly faster than that of RB-SBGMRES, we compared a broad selection of different matrices from the Matrix Market and presented a comparison of the overall condition number trend. We select

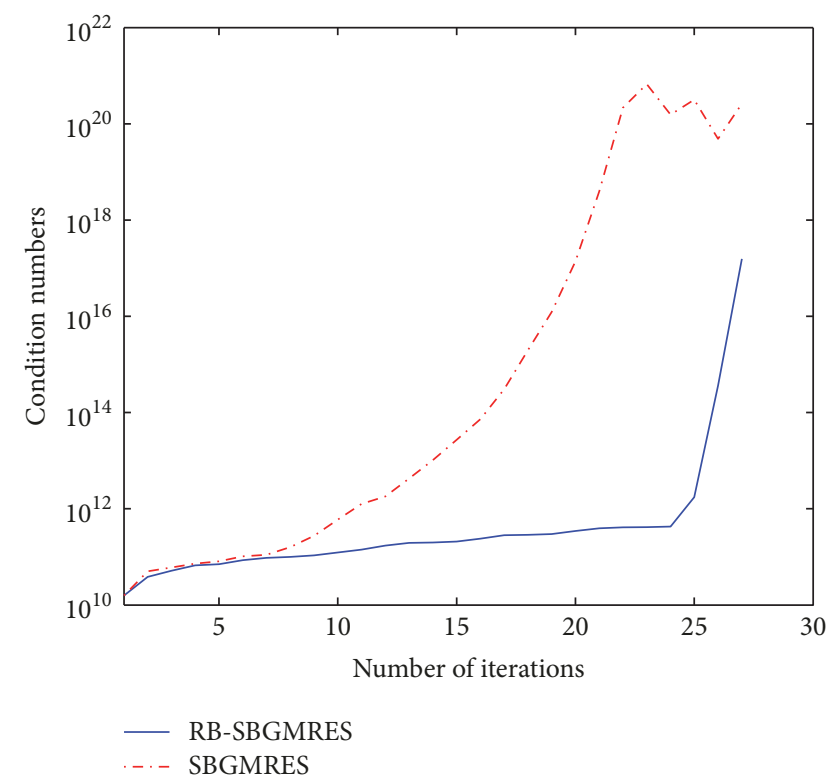

FIgURE 11: Example 4, the condition numbers versus the number of iterations.

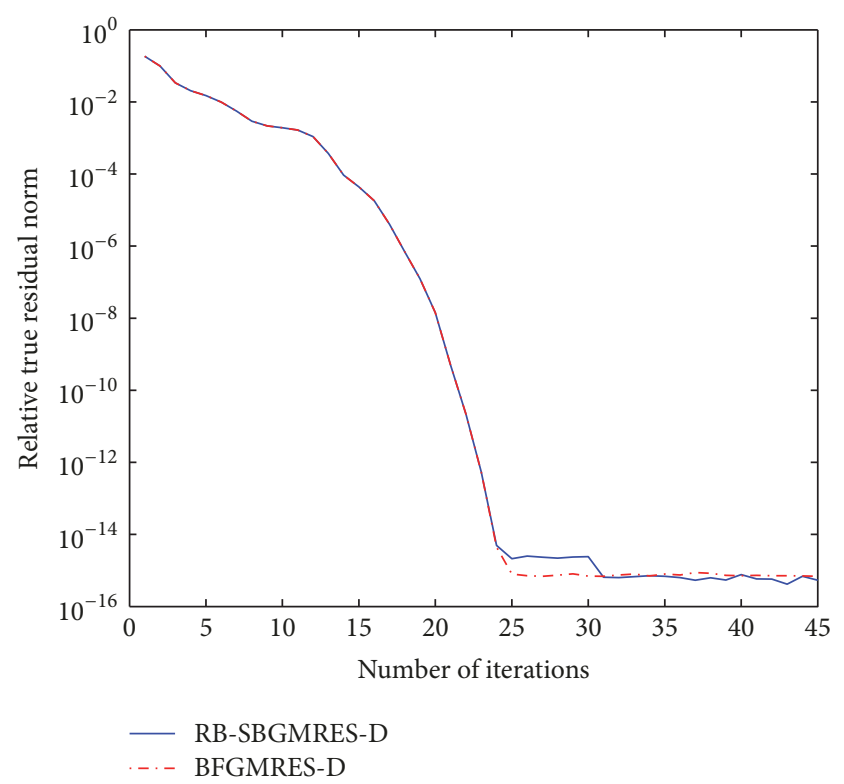

FIGURE 12: Example 4, the relative true residual norms versus the number of iterations.

matrices, randomly, and set the same convergence threshold for two methods. We compare the iterations required to converge for two methods. It is easy to see from Table 3 that the condition number of the matrix $\mathscr{U}_{m}$ of RB-SBGMRES is significantly smaller than that of SBGMRES and the number of iterations for the SBGMRES is slightly larger than that of RB-SBGMRES for most matrices.

\section{Conclusion}

In this paper, we have proposed a minimum residual method mathematically equivalent to the block GMRES method for 
TABLE 3

\begin{tabular}{|c|c|c|c|c|}
\hline \multirow{2}{*}{ Matrix } & \multicolumn{2}{|c|}{ RB-SBGMRES } & \multicolumn{2}{|r|}{ SBGMRES } \\
\hline & cond $\left(\mathscr{U}_{m}\right)$ & Iterations required to converge & cond $\left(\mathcal{U}_{m}\right)$ & Iterations required to converge \\
\hline $\mathrm{ck} 400$ & $1.5844 e+07$ & 99 & $1.0848 e+13$ & 99 \\
\hline nos3 & $1.8755 e+06$ & 123 & $6.6698 e+16$ & 135 \\
\hline dwa512 & $6.4465 e+06$ & 128 & $4.5023 e+13$ & 130 \\
\hline dwg961b & $5.1749 e+08$ & 238 & $6.7631 e+17$ & 242 \\
\hline dwt607 & $8.9814 e+05$ & 136 & $3.2494 e+13$ & 137 \\
\hline fidap001 & $7.1267 e+05$ & 53 & $2.3303 e+11$ & 53 \\
\hline fs5411 & $2.1648 e+04$ & 13 & $1.5152 e+17$ & 13 \\
\hline grel15 & $1.2874 e+04$ & 28 & $2.0989 e+11$ & 28 \\
\hline impcola & $2.8276 e+10$ & 51 & $4.1130 e+12$ & 51 \\
\hline jpwh991 & $1.2250 e+03$ & 61 & $2.3160 e+16$ & 65 \\
\hline $\operatorname{lns} 511$ & $1.3654 e+17$ & 70 & $1.4647 e+18$ & 86 \\
\hline mbeacxc & $5.8350 e+16$ & 112 & $1.1590 e+18$ & 112 \\
\hline $\operatorname{olm} 500$ & $2.6871 e+17$ & 68 & $1.3127 e+18$ & 68 \\
\hline orsirr2 & $5.1311 e+07$ & 221 & $6.6141 e+18$ & 222 \\
\hline pde900 & $3.3154 e+03$ & 94 & $8.9738 e+15$ & 98 \\
\hline pores1 & $2.0692 e+08$ & 7 & $3.3978 e+12$ & 7 \\
\hline qc324 & $9.5403 e+08$ & 81 & $1.6195 e+13$ & 92 \\
\hline qh768 & $1.5526 e+19$ & 30 & $4.3068 e+17$ & 30 \\
\hline $\mathrm{rdb} 450$ & $3.7541 e+11$ & 81 & $2.6783 e+17$ & 84 \\
\hline saylr1 & $1.6505 e+10$ & 59 & $1.0718 e+17$ & 62 \\
\hline sherman2 & $3.8156 e+15$ & 268 & $1.1791 e+19$ & 270 \\
\hline shl400 & $3.1199 e+10$ & 165 & $4.0589 e+10$ & 165 \\
\hline steam3 & $9.9777 e+07$ & 13 & $6.3224 e+19$ & 20 \\
\hline st 400 & $3.6364 e+16$ & 91 & $5.5214 e+17$ & 93 \\
\hline tols 340 & $7.7204 e+09$ & 48 & $1.9115 e+20$ & 49 \\
\hline utm300 & $7.5972 e+11$ & 73 & $1.5548 e+17$ & 73 \\
\hline curtis54 & $1.0376 e+04$ & 12 & $3.6682 e+06$ & 12 \\
\hline west 0132 & $3.6951 e+13$ & 32 & $2.9278 e+14$ & 32 \\
\hline nos5 & $1.8655 e+12$ & 117 & $4.3780 e+17$ & 119 \\
\hline nos7 & $3.6414 e+12$ & 130 & $4.2740 e+18$ & 183 \\
\hline
\end{tabular}

solving systems of linear equations with multiple right-hand sides. Numerical experiments show that, after some initial reduction, the relative true norms of residual SBGMRES may stagnate at a significantly higher level than that of RBSBGMRES. This difference is clearly caused by the choice of the basis $\mathscr{Z}_{m}$, which has an effect on the condition number of the matrix $\mathscr{U}_{m}$. Numerical experiments indicate that $\mathscr{U}_{m}$ of RB-SBGMRES remains better-conditioned than $\mathcal{U}_{m}$ of simpler block GMRES, which may become a very ill-conditioned triangular matrix. Since the coordinates of the correction $X_{m}-X_{0}$ in the basis $\mathscr{Z}_{m}$ are computed from (13), its error starts to diverge as $k\left(\mathcal{U}_{m}\right)$ grows and $X_{m}$ will become inaccurate. We see that the choice $\mathscr{X}_{m}=$ $\left[R_{0} /\left\|R_{0}\right\|_{F}, \ldots, R_{m-1} /\left\|R_{m-1}\right\|_{F}\right]$ has a better numerical performance. In comparison with the case of deflation, we consider a deflation strategy to detect the possible linear dependence of the residuals of the $s$ systems and a near rank deficiency occurring in the block Arnoldi procedure for RB-SBGMRES method, which was later called RB-SBGMRES-D. Numerical experiments show that the performances of RB-SBGMRES-D and BFGMRES-S are almost the same.

\section{Conflicts of Interest}

The authors declare that there are no conflicts of interest.

\section{Acknowledgments}

This work is supported by the National Natural Science Foundation of China under Grants 11701170 and 11501193, the Natural Science Foundation of Hunan Province under Grants 2017JJ3092 and 2017JJ2102, the Key Program of the Scientific Research Foundation from Education Bureau of Hunan Province under Grant 09A033, the Scientific Research Foundation of Education Bureau of Hunan Province for Outstanding Young Scholars in University under Grant 10B038, and the Young Core Teacher Foundation of Hunan Province in University. 


\section{References}

[1] Y. Saad, Iterative Methods for Sparse Linear Systems, SIAM, Philadelphia, PA, USA, 2nd edition, 2003.

[2] Y. Saad and M. H. Schultz, "GMRES: a generalized minimal residual algorithm for solving nonsymmetric linear systems," SIAM Journal on Scientific and Statistical Computing, vol. 7, no. 3, pp. 856-869, 1986.

[3] M. H. Gutknecht, "Block krylov space methods for linear systems with multiple right-hand sides: an introduction," in Modern Mathematical Models, Methods and Algorithms for Real World Systems, A. H. Siddiqi, I. S. Duff, and O. Christensen, Eds., pp. 420-447, Anamaya Publishers, New Delhi, India, 2007.

[4] V. Simoncini and E. Gallopoulos, "An iterative method for nonsymmetric systems with multiple right-hand sides," SIAM Journal on Scientific Computing, vol. 16, no. 4, pp. 917-933, 1995.

[5] V. Simoncini and E. Gallopoulos, "Convergence properties of block GMRES and matrix polynomials," Linear Algebra and its Applications, vol. 247, pp. 97-119, 1996.

[6] M. Robbé and M. Sadkane, "Exact and inexact breakdowns in the block GMRES method," Linear Algebra and Its Applications, vol. 419, no. 1, pp. 265-285, 2006.

[7] H. Calandra, S. Gratton, R. Lago, X. Vasseur, and L. M. Carvalho, "A modified block flexible GMRES method with deflation at each iteration for the solution of non-Hermitian linear systems with multiple right-hand sides," SIAM Journal on Scientific Computing, vol. 35, no. 5, pp. S345-S367, 2013.

[8] H. Liu and B. Zhong, "Simpler block GMRES for nonsymmetric systems with multiple right-hand sides," Electronic Transactions on Numerical Analysis, vol. 30, pp. 1-9, 2008.

[9] Matrix Market, http://math.nist.gov/matrixmarket.

[10] P. Jiránek, M. Rozložnk, and M. Gutknecht, "How to make simpler GMRES and GCR more stable," SIAM Journal on Matrix Analysis and Applications, vol. 30, no. 4, pp. 1483-1499, 2008.

[11] H. F. Walker and L. Zhou, "A simpler GMRES", Numerical Linear Algebra with Applications, vol. 1, no. 6, pp. 571-581, 1994. 


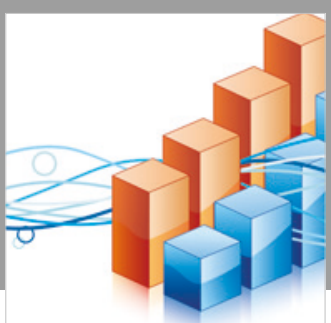

Advances in

Operations Research

\section{-n-m}
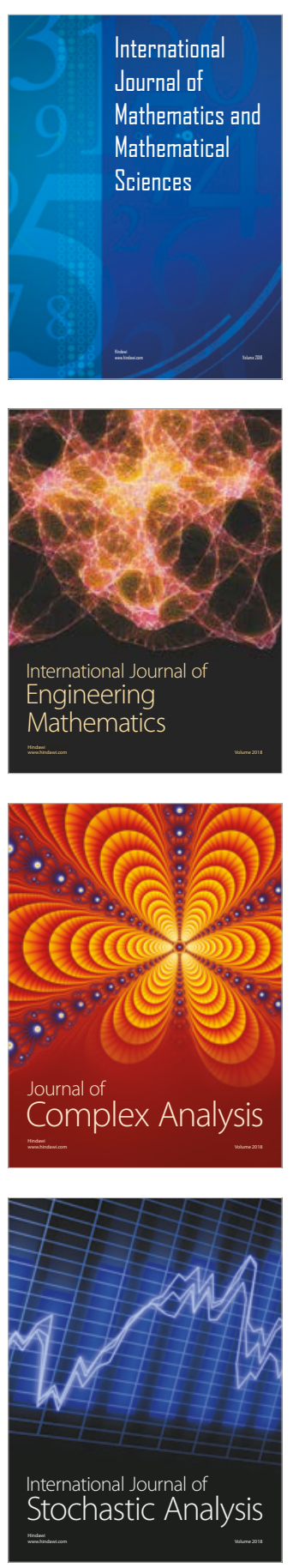
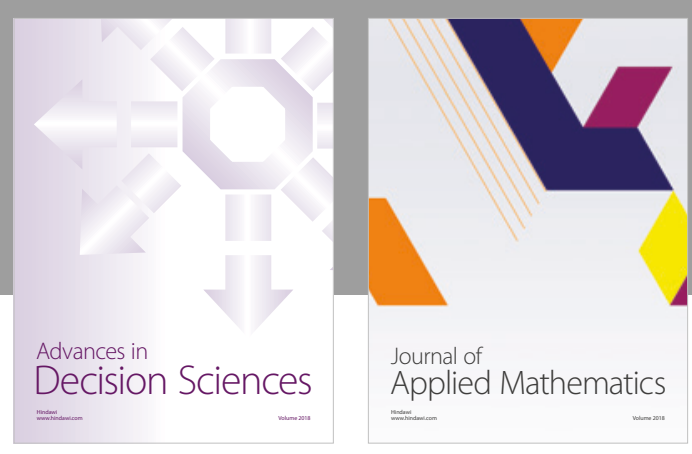

Journal of

Applied Mathematics
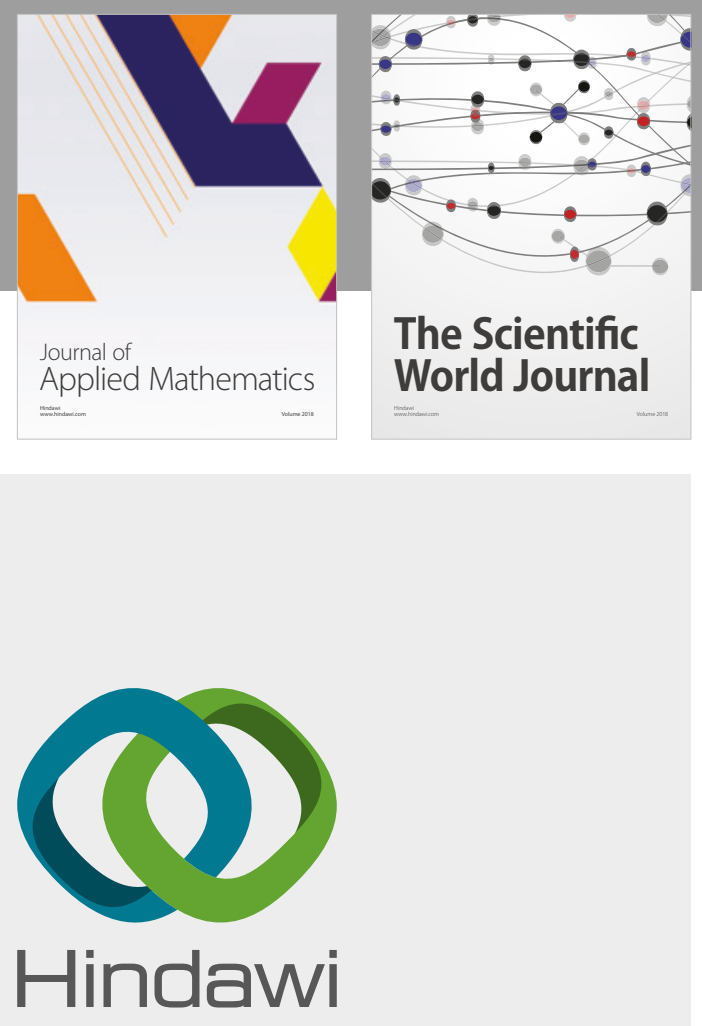

Submit your manuscripts at

www.hindawi.com

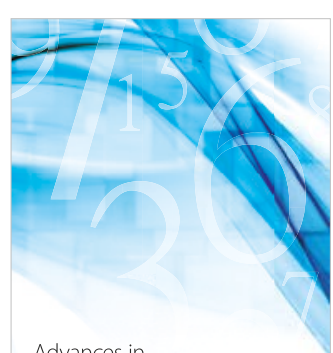

Advances in
Numerical Analysis
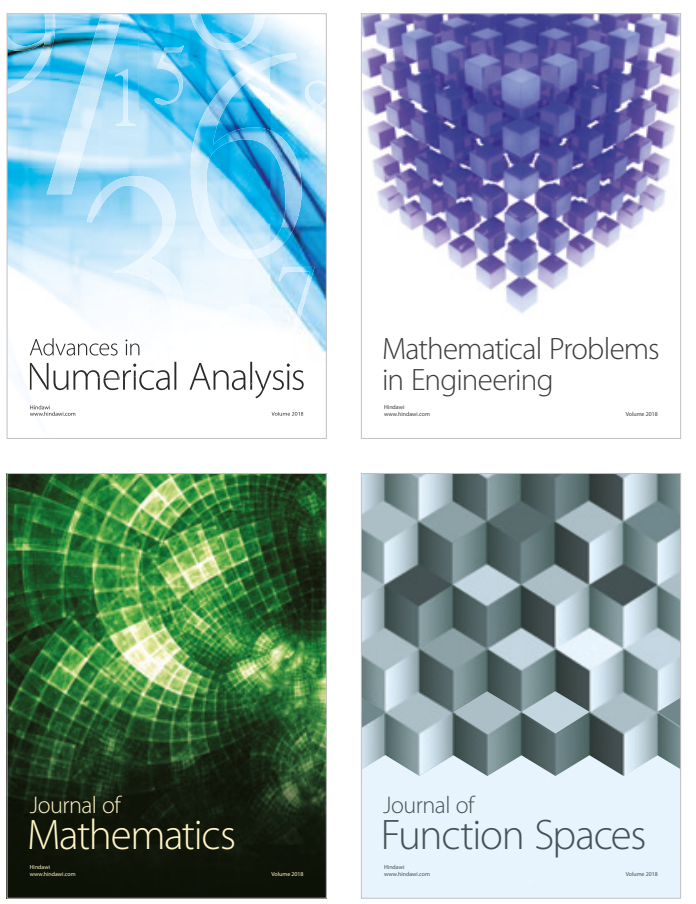

Mathematical Problems in Engineering

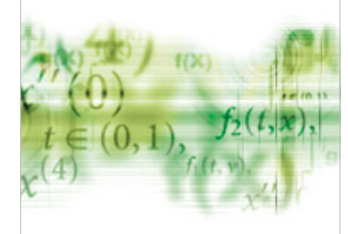

International Journal of

Differential Equations

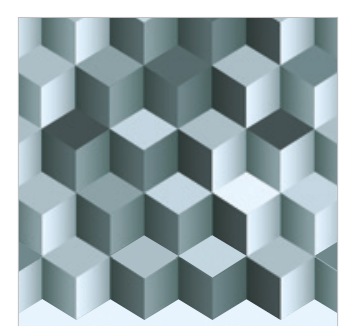

Journal of

Function Spaces

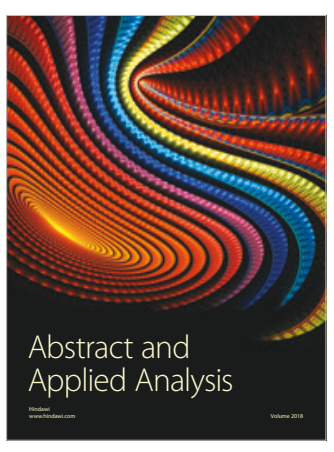

The Scientific

World Journal

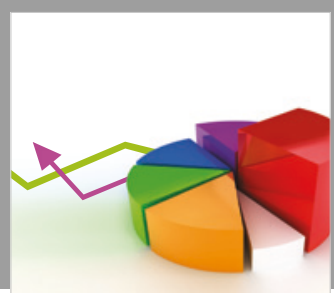

Journal of

Probability and Statistics
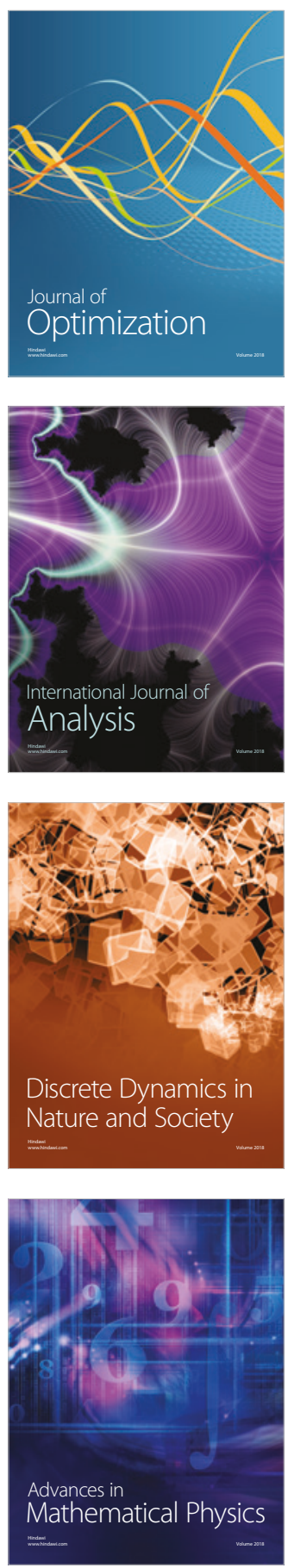